\title{
Modelling c-Abl Signalling in Activated Neutrophils: the Anti-inflammatory Effect of Seliciclib
}

\author{
Robert C. Jackson ${ }^{1 *}$ and Tomas Radivoyevitch ${ }^{2}$ \\ 'Pharmacometrics Ltd., Cambridge, UK \\ ${ }^{2}$ Department of Epidemiology and Biostatistics, Case Western Reserve University, Cleveland, Ohio, USA
}

\begin{abstract}
When mammalian tissues are infected by bacteria or fungi, inflammatory cytokines are released that cause circulating neutrophils to invade the infected tissue. The cytosolic tyrosine kinase, c-Abl, in these tissue neutrophils is activated by TNF $\alpha$. c-Abl then phosphorylates STAT transcription factors, which results in production of the antiapoptotic protein Mcl-1. The normally short-lived tissue neutrophils are then unable to enter apoptosis. c-Abl also causes release of reactive oxygen species (ROS) from the mitochondria of the activated neutrophils. These ROS, and ROS generated by NADPH oxidase, are bactericidal agents of the innate immune system. In some inflammatory diseases, such as chronic obstructive pulmonary disease (COPD), the invading neutrophils become permanently activated, and the resulting ROS overproduction causes severe tissue damage. The cyclin-dependent kinase inhibitor, seliciclib, blocks transcription through inhibition of cdk9. This results in a relatively rapid decline of antiapoptotic Mcl-1 transcripts in activated neutrophils, an increase in neutrophil apoptosis, and less ROS leakage and oxidative damage. We present here a model of neutrophil kinetics that simulates the principal pathways of c-Abl signalling and use it to explore possible treatment options for inflammatory lung disease.
\end{abstract}

Citation: Jackson RC, Radivoyevitch T. Modelling c-Abl Signalling in Activated Neutrophils: the Anti-inflammatory Effect of Seliciclib. Biodiscovery 2013; 7: 4; DOI: 10.7750/BioDiscovery.2013.7.4

Copyright: (C) 2013 Jackson. This is an open-access article distributed under the terms of the Creative Commons Attribution License, which permits unrestricted use, provided the original authors and source are credited.

Received: February 23, 2013; Accepted: March 29, 2013; Available online/Published: March 31, 2013

Keywords: c-Abl, Abelson gene, COPD, disease modelling, neutrophils, Mcl-1, ROS, seliciclib

Abbreviations: c-Abl, Abelson proto-oncogene; cdk9, cyclin-dependent kinase 9; CML, chronic myeloid leukaemia; COPD, chronic obstructive pulmonary disease; fu, fraction unaffected; ETC, electron transport chain; G-CSF, granulocyte colony-stimulating factor; GMCSF, granulocyte/ macrophage colony-stimulating factor; ICSBP, interferon consensus sequence binding protein; IL-3, interleukin 3; IL-8, interleukin 8; LPS, lipopolysaccharide; NAP-2, neutrophil activating peptide-2; PD, pharmacodynamics; PK, pharmacokinetics; ROS, reactive oxygen species; $\mathrm{SOD}$, superoxide dismutase; $\mathrm{TNF} \alpha$, tumour necrosis factor alpha.

"Corresponding Author: Robert C. Jackson, e-mail: rjackson1943@aol.com

Conflict of Interests: No potential conflict of interest was disclosed.

\section{Introduction}

Neutrophils represent the largest population of circulating white blood cells, and form an essential component of the innate immune system, the primitive form of cellular immunity that is believed to have predated the development of $\mathrm{T}$ cells and $\mathrm{B}$ cells in evolution, and which remains the first line of defence in mammals. In outline, the presence of bacterial cell wall components in tissues activates macrophages, which respond by releasing inflammatory cytokines. Interleukin 8 , in particular, attracts neutrophils to the site of infection [1]. Another cytokine, tumour necrosis factor alpha (TNF $\alpha$ ), was shown to be responsible for activation ("priming") 
of tissue neutrophils [2]. Neutrophil activation causes a number of changes, among which are inhibition of spontaneous apoptosis, and stimulation of release from mitochondria of reactive oxygen species (ROS). The gatekeeper molecule for neutrophil activation is the Abelson proto-oncogene (c-Abl), which is activated by $\mathrm{TNF} \alpha$ [3]. One of the chemokines produced by c-Abl is neutrophil-activating peptide-2 (NAP-2, also known as CXCL-7) which, as its name suggests, activates neutrophils [4]. The c-Abl protein is a cytosolic protein tyrosine kinase, which phosphorylates and activates the transcription factors STAT3 and STAT5. These STATs drive transcription of the anti-apoptotic proteins Mcl1 and Bcl- $\mathrm{X}_{\mathrm{L}}$, and also of cyclin D1 [4]. STAT3 also causes increases in fluxes into the electron transport chain (ETC) in mitochondria [5], with commensurate increases in electron back-pressure and thus ROS leakage from mitochondria. ROS arise when electrons fall into $\mathrm{O}_{2}$ at inappropriate sites of the ETC to produce the superoxide anion $\mathrm{O}_{2}$; the appropriate site on ETC complex IV gives $\mathrm{O}_{2} 4$ electrons and 4 protons, thus charging the proton gradient while splitting $\mathrm{O}_{2}$ into $2 \mathrm{H}_{2} \mathrm{O}$. ${ }^{-} \mathrm{O}_{2}$ then becomes hydrogen peroxide via superoxide dismutase (SOD) which has high activity in neutrophils. In unactivated neutrophils, low baseline levels of peroxide are removed by glutathione peroxidase, but in activated neutrophils, the capacity of this system is saturated, so that peroxide accumulates. This excess hydrogen peroxide then reacts with chloride anion to produce hypochlorite, which is bactericidal. This reaction is catalysed by the enzyme myeloperoxidase.

Formation of ROS is thus an essential part of the elimination of bacterial infection by neutrophils [6], but once the infection is eliminated it is essential that the activated neutrophils are removed, through apoptosis, because prolonged exposure to ROS can cause extensive normal tissue damage. If inflammatory cytokines are produced chronically, then c-Abl will remain activated, and primed tissue neutrophils will continue to produce ROS. This appears to be a major contributor to tissue damage resulting from inflammatory disease, including chronic obstructive pulmonary disease (COPD) [7-10]. COPD is the sixth largest cause of death worldwide. It is associated with smoking, and with inhalation of other irritants including environmental pollutants. The exact chain of events that results in a chronic inflammatory condition remains a matter of debate, but is generally attributed to inhalation of oxidative free radicals, stimulation of inflammatory cytokine production by inhaled irritants, and possibly an autoimmune contribution.

The process of neutrophil generation, trafficking, and activation is summarised in figure 1, which shows the activation of production of Mcl-1 (and thus inhibition of neutrophil apoptosis) by c-Abl. The other primary consequence of c-Abl activation, production of excessive ROS, is shown in more detail in figure 2. Several of the processes shown in figures 1 and 2 have been explored as targets for development of anti-inflammatory drugs.

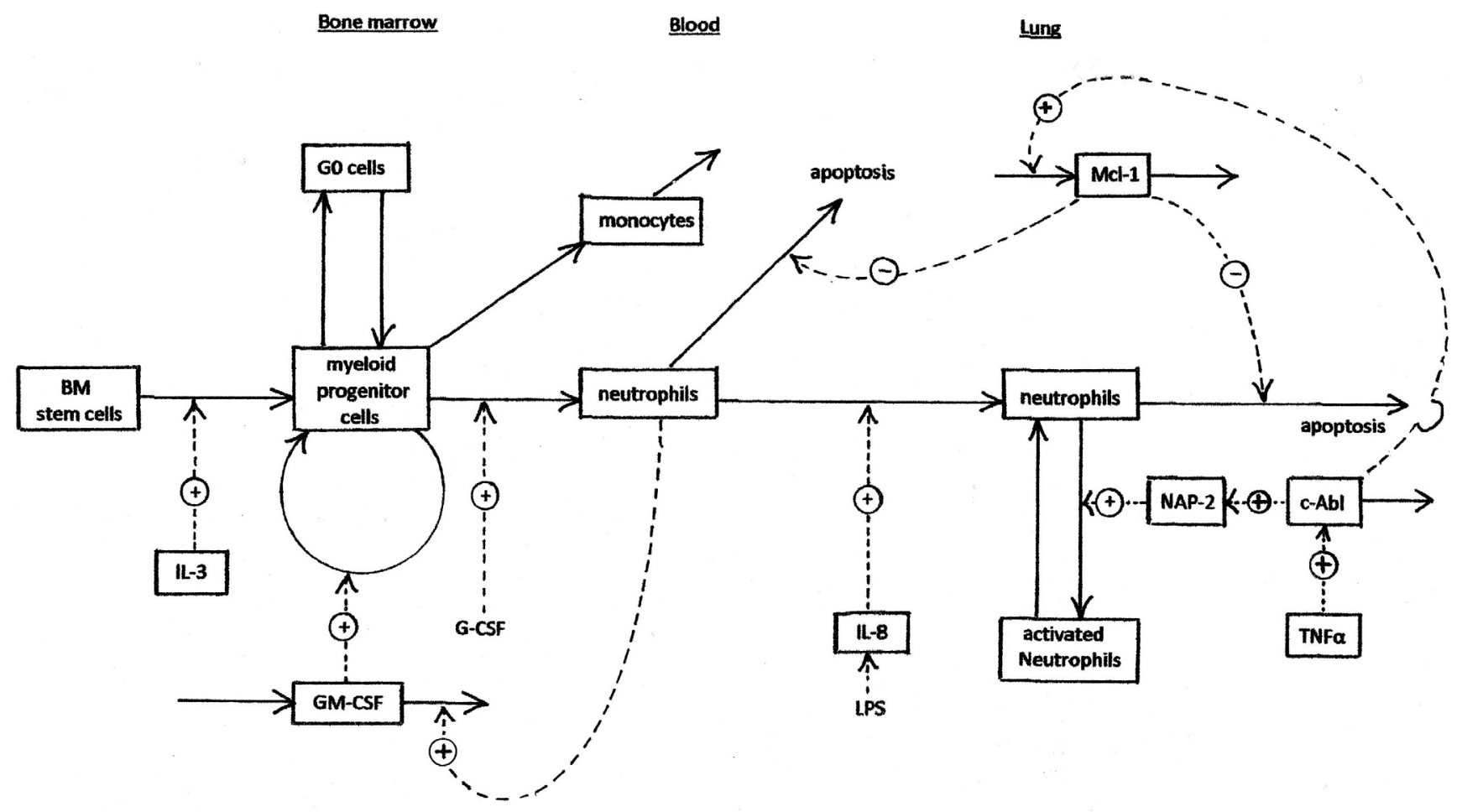

Figure 1. Neutrophil trafficking in the myeloN model. Components enclosed in rectangles are the 19 dynamic system state variables that are either cell populations or molecules. Other components are boundary conditions that do not change during the simulations. The model comprises 23 ordinary differential equations and 2 algebraic equations. Dotted lines indicate either activation $(+)$ or inhibition $(-)$. 
There has been particular interest in kinase inhibitors [11], anti-oxidants [12], and down-regulation of Mcl-1 $[13,14]$. An example of this last approach is the use of the cyclin-dependent kinase inhibitor, seliciclib, also known as R-roscovitine and CYC202 [13, 15]. Seliciclib is an inhibitor of $\operatorname{cdk} 9$, which phosphorylates and activates the C-terminal domain of RNA polymerase II [16]. This phosphorylation is required for efficient transcription, and its inhibition by seliciclib decreases many transcripts. However, when transcription ceases, the proteins that decline first are those that turn over most rapidly, one of which is Mcl-1. Seliciclib caused the decline of Mcl1 within 90 minutes, its almost complete disappearance within 3 hours, and continuing absence still at 24 hours [17].

The kinetics of signalling pathways are complicated by multiple positive and negative feedback loops, cross-talk between the different pathways, action across multiple intracellular compartments, and oscillatory dynamics. Understanding these complex kinetics has been facilitated by constructing computer models of signalling networks $[18,19]$. We here describe a model of c-Abl signalling in neutrophils. The approach adopted has been to build upon earlier models of myeloid cell maturation [20] by adding elements of the c-Abl signalling pathway, including all the processes shown in figures 1 and 2. Other published models that have guided aspects of our model design are those of Zhuge et al.[21], Brookes et al. [22] and Scholz et al. [23]. Our model could serve as a tool for exploring the comparative activity of different therapeutic approaches, to explore potential synthetic lethalities resulting from c-Abl activation, and to design optimal drug combination protocols.

\section{Methods}

\section{Description of the myeloN Model}

The scope of the model is shown in Figures 1 and 2 . Bone marrow stem cells and myeloid progenitor cells are able to replicate. They remain localised in bone marrow. Neutrophils and monocytes do not replicate. The equations of the model are set out in the program listing (supplementary material). The system is basically a Bayesian prior belief model that is valid to the extent that it produces reasonable responses.

\section{Control mechanisms of the model}

1. The proliferating population of myeloid progenitor cells has a kinetic reserve of G0 cells. These cells can be brought back into cycle in response to depletion of the total proliferating compartment, e.g. after myelosuppressive chemotherapy (37).

2. GM-CSF is increased when the number of circulating neutrophils is low (e.g. after chemotherapy). The model treats this as binding of GM-CSF by circulating neutrophils (in which it has no effect), thus lowering the steady-state level of GM-CSF. This is a major homeostatic mechanism.

3. Interleukin 8 is stimulated by lipopolysaccharide (LPS), e.g. after infection, resulting in increased trafficking of neutrophils from blood into tissues. Infection also causes increased TNF $\alpha$.

4. c-Abl is activated by TNF $\alpha$ resulting from infection or inflammation. c-Abl then phosphorylates STAT5 and causes neutrophil activation by up-regulation of Mcl-1 and Bcl-x. These proteins are inhibitors of apoptosis.

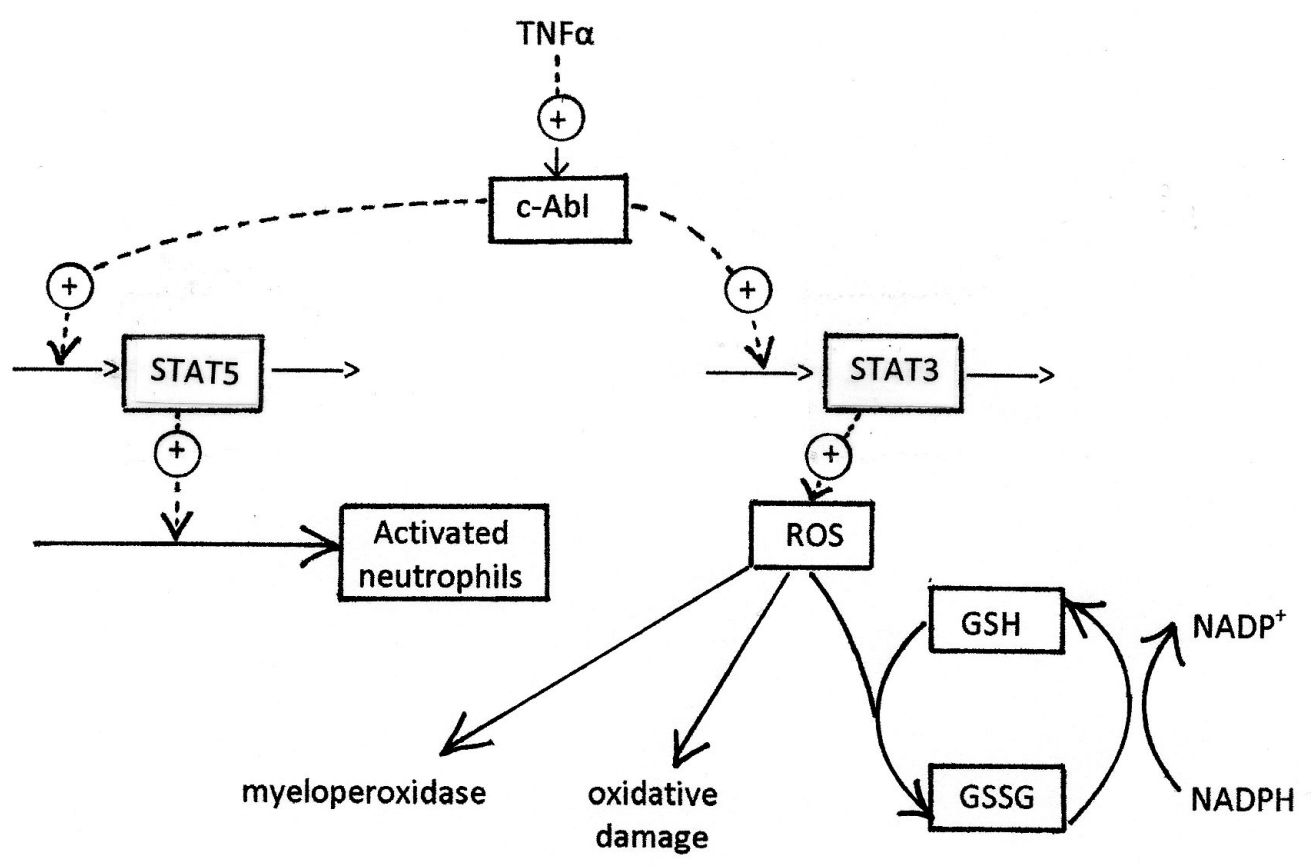

Figure 2. The two-pronged effect of c-Abl activation. See notes to figure 1. 
5. Interferon- $\alpha$ phosphorylates and dimerises STAT5, which drives transcription of ICSBP, which represses transcription of anti-apoptotic proteins.

6. c-Abl activates production of IL-3 and GM-CSF.

\section{Sites of drug action}

Nine of the rate processes are subject to inhibition by drugs. The drug effects that are modelled are: (1) DNA cross-linking, which inhibits cell proliferation (e.g. busulphan); (2) Inhibition of GM-CSF (e.g. MOR103); (3) Inhibitors of c-Abl tyrosine kinase (e.g. imatinib); (4) inhibition of CXCR1 and CXCR2 cytokine receptors (e.g. SB272844); (5) DNA synthesis inhibitors (e.g. cytarabine); (6) Inhibitors of Mcl-1 transcription (e.g. seliciclib); (7) TNF $\alpha$ antagonists (e.g. infliximab); (8) Induction of ICSB transcription (e.g. $\alpha$-interferon); (9) Anti-oxidants (e.g. ascorbate). Currently, only constant concentrations of drugs and cytokines are modelled.

\section{Modelling infection}

Infection is treated as an instantaneous event occurring at zero time. LPS, a bacterial cell wall component, induces a range of cytokines. IL-3, IL-8 and TNF $\alpha$ are explicitly simulated by the model. G-CSF, which is produced by activated macrophages, is also modelled. IL- 8 then triggers migration of neutrophils from plasma into the infected tissue, while TNF $\alpha$ activates c-Abl. c-Abl stimulates the release of ROS from the mitochondrial electron transport chain. Other ROS sources were not modelled, though they could be. Regardless of their source, ROS interact with myeloperoxidase to release hypochlorite, which is bactericidal. The model then assumes that bacteria are destroyed by ROS, and that this effect follows a Hill equation. The saturation function given by the Hill equation is interpreted as a fraction of the maximum antibacterial effect, expressed as log kill per hour:

$$
\mathrm{bk}=\mathrm{bkmax}^{*} \operatorname{ROS}^{\mathrm{n}} /\left(\mathrm{K}_{\mathrm{m}}+\mathrm{ROS}^{\mathrm{n}}\right) \text {. }
$$

Then the bacterial count at time $\mathrm{t}+\mathrm{dt}=$ bacteria $_{\mathrm{t}}$ / $\left(10^{\mathrm{bk}} \mathrm{dt}\right)$. LPS, IL8 and TNF $\alpha$ change in proportion with the bacterial count.

\section{Modelling inflammation}

Inflammation, specifically COPD, is modelled by assuming a constant level of TNF $\alpha$, which results in permanent activation of c-Abl. Only cells in the tissue are affected, and no c-Abl activation is assumed in blood or bone marrow.

\section{Programming}

The model is implemented in the programming language $\mathrm{R}$ [38]. Ordinary differential equations are solved using the $\mathrm{R}$ package deSolve. The equations of the model are included in the supplementary material and the program listing may be obtained from an R package called myelo that we are developing on github to house myelopoiesisrelated models; see http://epbi-radivot.cwru.edu/myelo/ myelo.html for installation directions and once installed, see the help page of the function rcj12() which provides the right hand side of the model's differential equations used by deSolve.

Parameter values are listed in the supplementary material. Parameter estimates are based upon literature data $[1,2$, $4,7-9,13-16,19-23]$. Initial conditions were chosen to represent cell numbers in either the unstimulated steady state or, for simulation of anti-inflammatory drug effects, the untreated inflamed state in presence of activated macrophages.

\section{Results}

To demonstrate that the model predicted reasonable values in the absence of infection, inflammation, or drugs, we first simulated the unperturbed steady-state. Using the default starting conditions, with no activation of c-Abl, the system was stable (see supplementary material). Output from selected simulations is shown in the main text, and all simulations are summarized in the supplementary material. The modelled circulating neutrophil count was about $4.6 \mathrm{million} / \mathrm{ml}$ blood, and the steady-state count of tissue-infiltrating neutrophils was $75,000 / \mathrm{gm}$ tissue. Only $0.1 \%$ of these were activated in the absence of $\mathrm{c}-\mathrm{Abl}$ stimulation. The calculated mean residence time of neutrophils in plasma was 4.7 days. A low steady state level of ROS was well within the capacity of neutrophil glutathione peroxidase to remove [24]. Units of cycling progenitor cells and of G0 progenitor cells are cells/gm of bone marrow.

Interleukin 8 (IL-8) is produced by tissue macrophages, which are activated in the presence of bacterial or fungal infection, or inflammation. In the absence of infection or immune stimulation, tissue levels of IL-8 are very low. Simulation 2 modelled the effect of continuous exposure to a high concentration $(50 \mathrm{nM})$ of IL- 8 . This treatment resulted in an increase in tissue neutrophils of about 2.6fold, peaking at about 22 hours. An increase in activated tissue neutrophils was also predicted, of about 2.4-fold, peaking at $36 \mathrm{hr}$. There was a marked decline in the blood neutrophil count, correlating with the migration of these cells into tissues. The decreased blood neutrophil count triggered an increased proliferation of progenitor cells, which in turn began to restore the blood neutrophil count. ROS increased by about 2-fold, reflecting the increase in activated tissue neutrophils.

After treatment for 9 days with a maximum tolerated concentration of the interleukin 8 antagonist, SB272844, tissue neutrophils reached a nadir of $11 \%$ of the normal value. Circulating neutrophils increased (simulation 3). 
The level of ROS production decreased to about $50 \%$ control. These predictions are consistent with the IL-8 antagonist's mechanism of inhibiting trafficking of neutrophils from blood into tissues.

A subsequent study (simulation 4) modelled the effect of IL-8 $(50 \mathrm{nM})$ in presence of its antagonist, SB272844 $(5 \mu \mathrm{M})$. As seen by comparing simulation 4 with simulation 3, the increase in tissue neutrophils caused by IL-8 was completely prevented. No increase in activated neutrophils was seen, in contrast to the 2.4-fold increase predicted in absence of SB272844. The increase in ROS driven by IL- 8 was also completely blocked.

MOR103 is a monoclonal antibody that binds to GMCSF and thus prevents its interaction with the GM-CSF receptor [25]. It is under clinical evaluation for treatment of rheumatoid arthritis, a disease where tissue damage is caused by invasion of activated neutrophils. Simulation 5 modelled the effect of $10 \mathrm{nM}$ MOR103. Note the rapid depletion of myeloid progenitor cells (labelled "CFUGM" in the corresponding table of the supplement). The model predicted a decrease of $74 \%$ in activated neutrophils over 10 days.

Simulation 6 examined the effect of increasing G-CSF to 5-fold the normal level. Over the first few days of treatment the model predicted an $11 \%$ increase in circulating neutrophils, coupled with a $46 \%$ decrease in myeloid progenitor cells. This relatively muted response presumably reflects the strong homeostatic regulation of circulating neutrophils, which cause feedback inhibition of the proliferation of progenitor cells [21]. A more marked effect was predicted for increasing the level of GM-CSF to $12.5 \mathrm{x}$ the normal value for 12 days (simulation 7). Myeloid progenitor cells increased 3.9-fold by 12 days, and circulating neutrophils increased 2.6-fold. Tissue neutrophils increased 2.2-fold. The cytokinetic reserve of G0 progenitor cells was predicted to show a small relative increase from $8 \%$ to $9.3 \%$ under these conditions.

In simulation 8 , a bacterial infection $\left(10^{6}\right.$ cells/ gm tissue) is assumed to result in the presence of lipo-polysaccharide (LPS) in tissues. This activates macrophages, which release $\mathrm{TNF} \alpha$, and activation of c-Abl results. This causes a strong antibacterial response. By ten days, $99.9 \%+(5.9 \operatorname{logs})$ of the bacteria have been eliminated, and the ROS level (which peaks at $84 \mathrm{~h}$ ) is returning to normal. Following an initial dip in circulating neutrophils, as they migrate into the infected tissues, a moderate leucocytosis is seen, with circulating neutrophils peaking at $255 \%$ normal at $184 \mathrm{~h}$.

The antibacterial response was dependent on the size of the bacterial challenge. Simulation 9 shows a bacterial challenge of $10^{5}$ cells/gm tissue. By 10 days, the bacteria were completely eliminated. ROS peaked at $6.0 \mathrm{x}$ control at $60 \mathrm{~h}$. Circulating neutrophils increased $21 \%$, peaking at $240 \mathrm{~h}$. After a very large bacterial challenge of $10^{7}$ cells/ gm tissue (simulation 10), ROS reached levels almost 50x baseline. Bacterial kill was about 6 logs, and circulating neutrophils peaked at 6.5 -fold control, at $230 \mathrm{~h}$. At these high levels of ROS accumulation, the redox buffer system of the cell is saturated, and significant amounts of oxidised glutathione appear.

In bacterial infection, the activation of c-Abl is driven, indirectly, by bacterial cell wall LPS. As the infection is eliminated, production of ROS tapers off. The antibacterial response is self-limited, so that oxidative damage to host tissues is minimal. The situation is different in inflammatory disease, where inflammatory cytokines, including TNF $\alpha$, are produced continually, so that c-Abl is permanently activated. We modelled this situation by setting TNF $\alpha$ to a constant level of $5 \mathrm{nM}$ (simulation 11). This resulted in c-Abl activation $(0-6.34$ in $48 \mathrm{~h})$, activation of tissue neutrophils (5.6-fold in $48 \mathrm{~h}$ ), Mcl-1 (7.1-fold), and ROS (3.8-fold, and still rising at $48 \mathrm{~h}$ ). ROS production eventually levelled off at more than 50 -fold the baseline value, a level likely to cause significant tissue damage. Circulating neutrophils increased moderately $(40 \%$ at $48 \mathrm{~h})$, and tissue neutrophils increased $61 \%$, presumably because of decreased apoptosis. This situation may be regarded as a first approximation model of COPD, for the purposes of in silico drug screening.

If the primary cause of neutrophil activation in COPD is TNF $\alpha$, one way to prevent c-Abl activation may be to treat with the anti-TNF antibody, infliximab. Simulation 12 summarises the result of simultaneous administration of TNF $\alpha$ and infliximab. Infliximab (5 nM) inhibited the response to $\mathrm{TNF} \alpha$, decreasing the effect on neutrophil activation by $81 \%$ at $48 \mathrm{~h}$, the effect on ROS by $75 \%$, and the effect on Mcl-1 by $83 \%$.

There have been several studies of the effects of antioxidants, including ascorbate, on COPD, with some preliminary, but encouraging, results on biomarkers of oxidative stress [7-10]. The myeloN model assumes, as a default value, that human plasma contains $7 \mathrm{mM}$ ascorbate. Simulation 13 calculated the baseline ROS production in complete absence of anti-oxidants, and simulation 14 calculated ROS production at $7 \mathrm{mM}$ ascorbate. ROS production at this mean physiological level was $74 \%$ of the value in absence of anti-oxidants, showing only a modest protective effect. Increasing ascorbate to $30 \mathrm{mM}$ (a concentration that can be achieved by taking very high doses of oral ascorbic acid) decreased ROS production by $56 \%$ (simulation 15 ). A more relevant question is the extent to which high doses of ascorbate can decrease the greatly elevated levels of ROS found in COPD. Simulation 16 allows the ROS production to reach $95 \%$ of its steadystate value after addition of $5 \mathrm{nM} \mathrm{TNF} \alpha$, then adds 30 $\mathrm{mM}$ ascorbate at $480 \mathrm{~h}$. ROS production fell by $75 \%$, but was still ten-fold higher than the normal level, suggesting that more powerful antioxidants might be beneficial for 
treatment of COPD.

Because of evidence that the primary factor involved in neutrophil activation is the anti-apoptotic protein Mcl1 [14], there has been interest in inhibition or downregulation of Mcl-1 as an approach to treatment of COPD. The Mcl-1 protein and its mRNA both have short turnover times, so inhibition of protein synthesis, or inhibition of transcription of mRNA, although not inherently selective, can cause a disproportionate decrease in Mcl-1. Inhibitors of cdk9, such as seliciclib [16, 17] inhibit transcription by blocking phosphorylation of the C-terminal domain of RNA polymerase II. Seliciclib has shown activity in several animal models of inflammatory disease, including inflammatory lung disease [13-15]. Simulation 17 modelled the effect of a constant concentration of $5 \mu \mathrm{M}$ seliciclib on a model of COPD in which c-Abl is kept activated by chronic exposure to $0.5 \mathrm{nM}$ TNF $\alpha$. Mcl-1 was down-regulated by $94 \%$, and this was accompanied by an $83 \%$ decrease in the number of activated neutrophils and a $70 \%$ decrease in ROS. Seliciclib was predicted to have a moderately neutropenic effect, but this was much less than its effect on ROS. Modelling the seliciclib dose-response relationship showed that ROS could be inhibited by up to $87 \%$, but circulating neutrophils did not drop below $50 \%$ of control, even at very high seliciclib concentrations (Figure 3).

Chronic myeloid leukaemia, CML, is a form of leukaemia in which a $\mathrm{t}(9: 22)$ chromosomal translocation produces a fusion protein in which a c-Abl domain is fused to the protein product of the Bcr gene, and as a result the Abl domain is constitutively activated [26-28]. The BcrAbl kinase inhibitor, imatinib (Glivec ${ }^{\circledR}$ ) has been used very successfully to treat CML $[29,30]$. Imatinib and its analogues do not appear to distinguish between the BcrAbl kinase domain and that of normal c-Abl, suggesting that imatinib could be used to treat COPD. There are no published reports of imatinib being used to treat COPD, though other kinase inhibitors have been explored for this indication [11]. Simulation 18 attempted to predict the effect of a high concentration $(300 \mathrm{nM})$ of imatinib on COPD. The level of ROS was decreased by $90 \%$. The tissue count of activated neutrophils was decreased by $93 \%$, and Mcl-1 activity was lowered $65 \%$.

The fact that both imatinib and seliciclib were predicted to have strong activity against COPD, and by different mechanisms, suggested that it would be interesting to predict the efficacy of combinations of the two agents. The myeloN model was used to simulate effects of combinations of imatinib (at concentrations ranging from $10-120 \mathrm{nM})$ and seliciclib $(0.3-70 \mu \mathrm{M})$. Results were analysed by fitting the three-dimensional dose-response surface to the equation of Greco et al. [31]. This equation describes the response of the system to each drug as a Hill function, and includes an additional interaction parameter, $\alpha$. If $\alpha=0$, the two agents are additive; $\alpha<0$ implies antagonism, and $\alpha>0$ indicates synergism. The Greco equation thus describes the degree of inhibition of a system in presence of two drugs in terms of 5 parameters: $\mathrm{IC}_{50}$ and slope of the dose-response curve for each drug individually, and $\alpha$. In descriptions of inhibition kinetics, it is conventional to describe the amount of inhibition as fraction affected $\left(f_{a}\right)$, e.g. for a system that is $35 \%$ inhibited, $\mathrm{f}_{\mathrm{a}}=0.35$. The fraction unaffected, $\mathrm{f}_{\mathrm{u}}$, is then given by $\mathrm{f}_{\mathrm{u}}=$ $1-f_{a}$. For the combination of imatinib + seliciclib, using ROS as endpoint, the myeloN model predicted an $\alpha$ value of 3.70 , corresponding to moderate synergy. Presenting

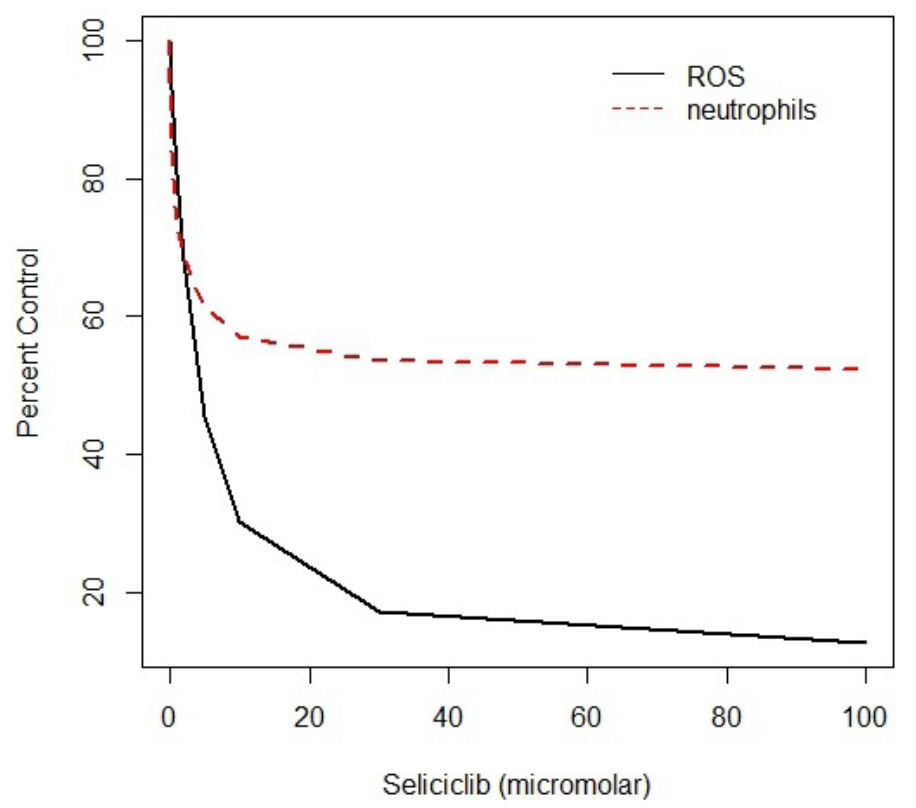

Figure 3. Dose-response curve for the effect of seliciclib on ROS and circulating neutrophils. The myeloN model was used to simulate effects of seliciclib concentrations of 0.3 to $100 \mu \mathrm{M}$ on the steady state. 
the interaction as an isobol plot (Figure 4) shows the concave shape characteristic of a synergistic interaction. Isobols show the degree of drug interaction at levels required to produce a fixed endpoint - in this case an $\mathrm{IC}_{50}$.

Figure 5 shows the drug interaction as a 3 -dimensional response surface. This form of data presentation has the advantage that it covers the entire range of concentrations for both drugs. The complex shape of this plot reflects the fact that the slope of the Hill function for imatinib is much steeper than for seliciclib, creating an asymmetry in the shape of the surface. The $\mathrm{R}$ programming language, which was used to plot this figure, makes it possible to rotate the diagram on a computer screen, giving a better impression of the details of the response surface.

Another popular method of depicting drug interaction data is the median effect plot of Chou and Talalay [32]. This method measures the effect of mixtures of the two drugs, at various concentrations, but at a fixed ratio. The sum of the amounts of each drug, as a fraction of its median effective dose, is termed the combination index (CI). For example, if achieving $50 \%$ inhibition of a system by a combination of two drugs required $50 \%$ of the $\mathrm{IC}_{50}$ level of the first drug and $70 \%$ of the $\mathrm{IC}_{50}$ level of the second drug, then $\mathrm{CI}=0.5+0.7=1.2$. Unlike Greco's alpha parameter,

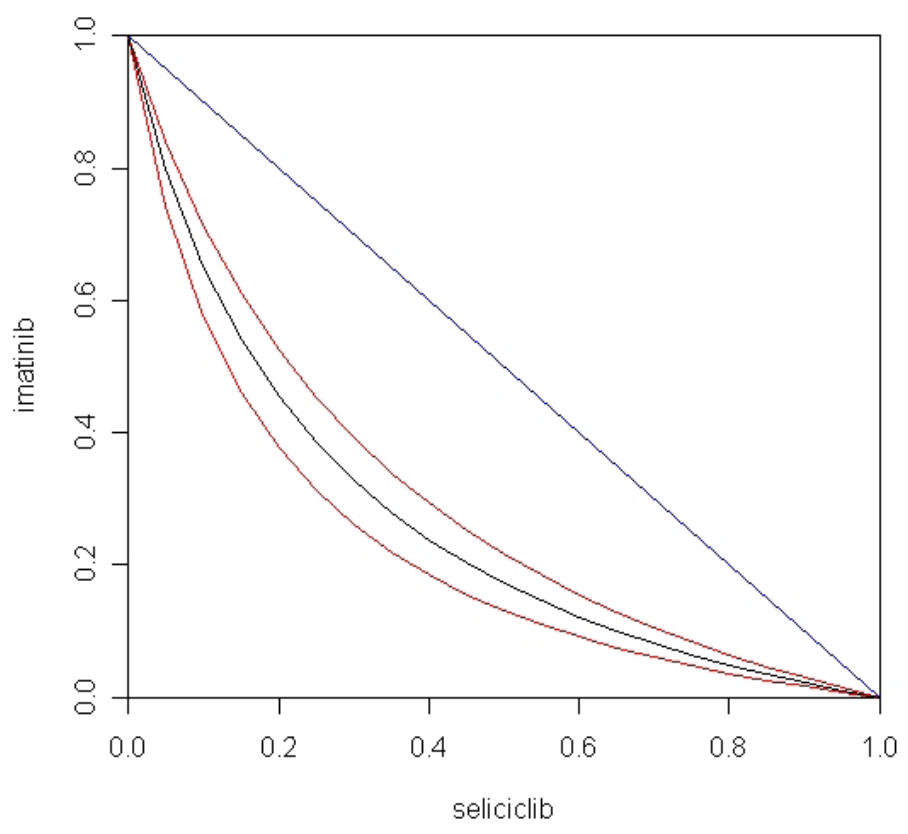

Figure 4. Isobol plot for the imatinib + seliciclib combination. The diagonal shows the line of additivity. The concave shape of the isobol indicates synergy; the lines above and below the isobol are the upper and lower $95 \%$ confidence intervals.

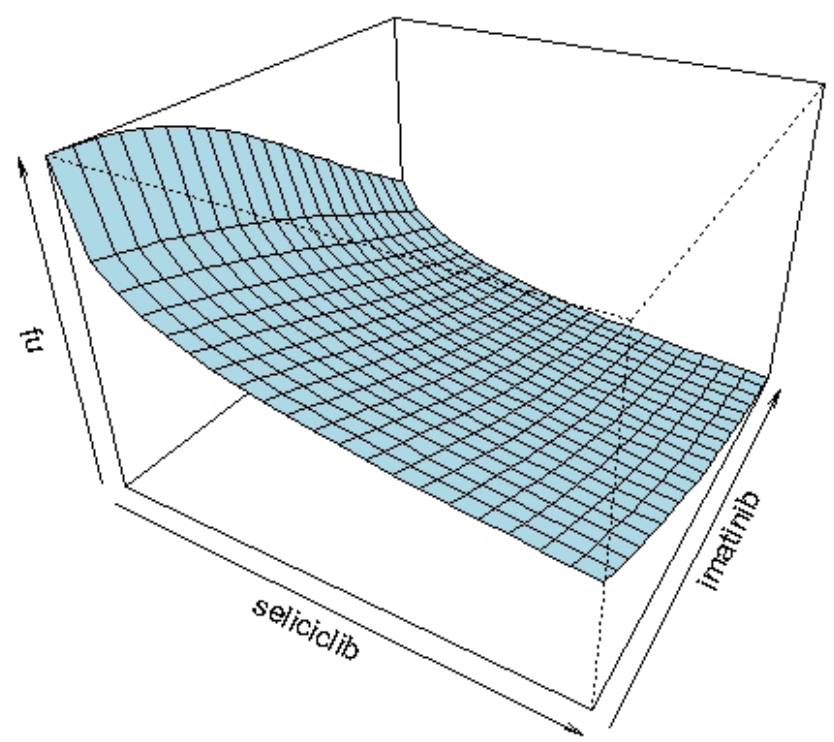

Figure 5. 3-Dimensional response surface for the imatinib + seliciclib combination. Arrows on the $\mathrm{x}$ and $\mathrm{y}$ axes indicate directions of increasing drug concentration. $\mathrm{fu}=$ fraction unaffected. 
$\mathrm{CI}$ is not constant over the concentration range, but varies with the degree of inhibition. Values of $\mathrm{CI}=1$ correspond to additivity, $\mathrm{CI}>1$ corresponds to antagonism, and CI $<1$ to synergism. Figure 6 shows CI versus $\mathrm{f}_{\mathrm{a}}$ (fraction affected) for the imatinib + seliciclib combination. The combination was predicted to be synergistic over most of the concentration range, and approximately additive at high degrees of inhibition.

The combination of seliciclib and ascorbate was also modelled, using inhibition of ROS as endpoint. The calculated alpha parameter was -0.023 , not significantly different from zero, indicating an additive interaction. The isobol plot for this combination is shown in Figure 7.

Finally, the myeloN program was used to model the interaction between seliciclib and SB272844. The rationale for this combination was that seliciclib triggers apoptosis of activated neutrophils, whereas SB272844 blocks trafficking of circulating neutrophils into tissues. Simulation 19 shows the effect of $1.34 \mu \mathrm{M}$ seliciclib. Based upon the value of ROS at $480 \mathrm{~h}$, the degree of inhibition is $50.0 \%$, i.e. the fraction unaffected $(\mathrm{fu})=0.5$.

Simulation 20 shows the effect of $2.43 \mu \mathrm{M} \mathrm{SB} 272844$.

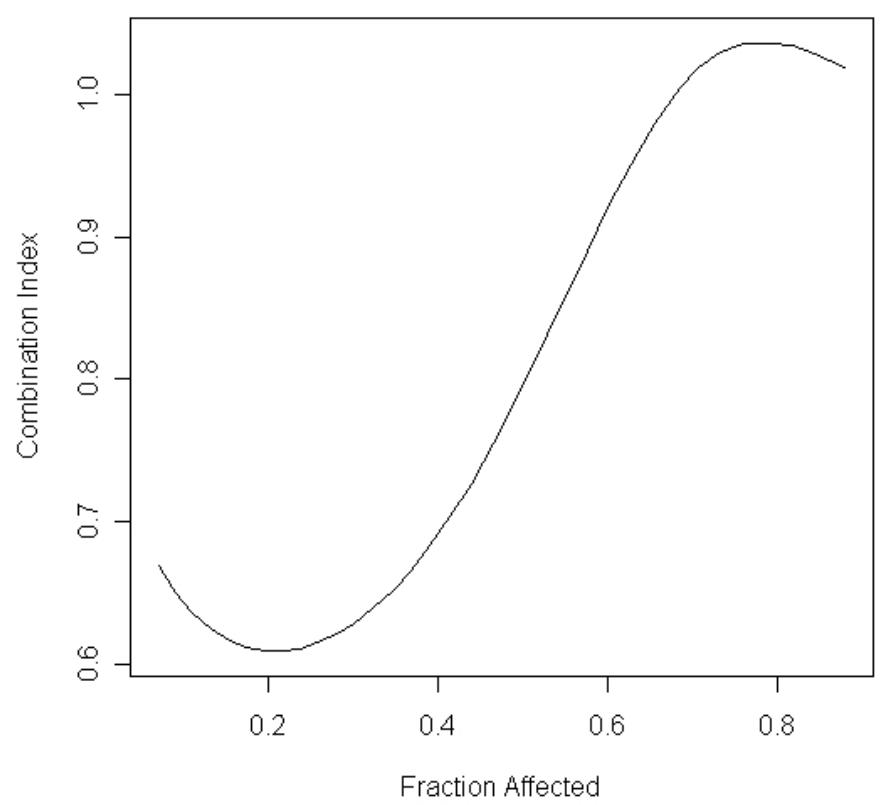

Figure 6. Combination index values for the imatinib + seliciclib combination. Predicted values of ROS calculated by the myeloN program were analysed by the method of Chou and Talalay [32].

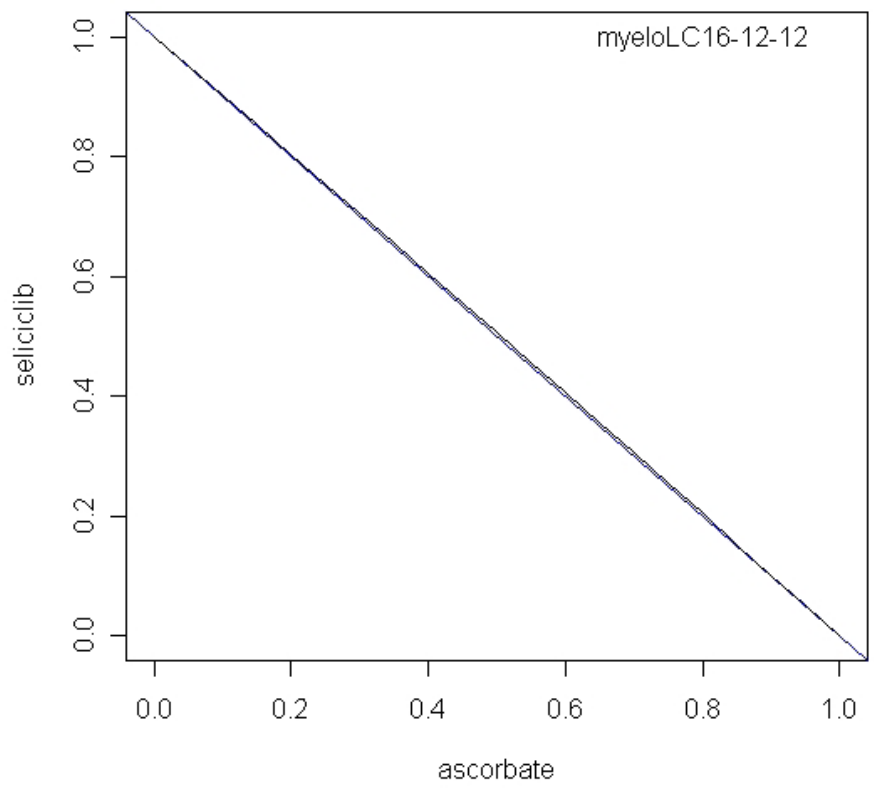

Figure 7. Isobol plot for the ascorbate + seliciclib combination. The diagonal shows the line of additivity. The isobol line overlays the additivity line, indicating that the combination does not differ significantly from additivity. 
Over $480 \mathrm{~h}$, this treatment reduces leakage of ROS by $50 \%$, i.e. $\mathrm{fu}=0.5$. Simulation 21 shows the combined effect of $1.34 \mu \mathrm{M}$ seliciclib plus $2.43 \mu \mathrm{M}$ SB272844. ROS leakage is inhibited by $87.6 \%$ by 480 h, i.e. $\mathrm{fu}=$ 0.124 . If the two drugs act independently, as defined by Bliss [32], the fraction unaffected by the combination would be $0.5 \times 0.5=0.25$. Since the actual value is less than half of this, the combination is synergistic, as defined by Bliss. Using the response surface approach of Greco et al. [31,33] gave an alpha value of $8.72(95 \%$ CL 6.58 - 12.21) corresponding to strong synergism. The isobol for the combination is shown in Figure 8, and is markedly concave. These predictions suggest that the combination of a drug that down-regulates Mcl1 with a drug that inhibits neutrophil trafficking merits experimental evaluation.

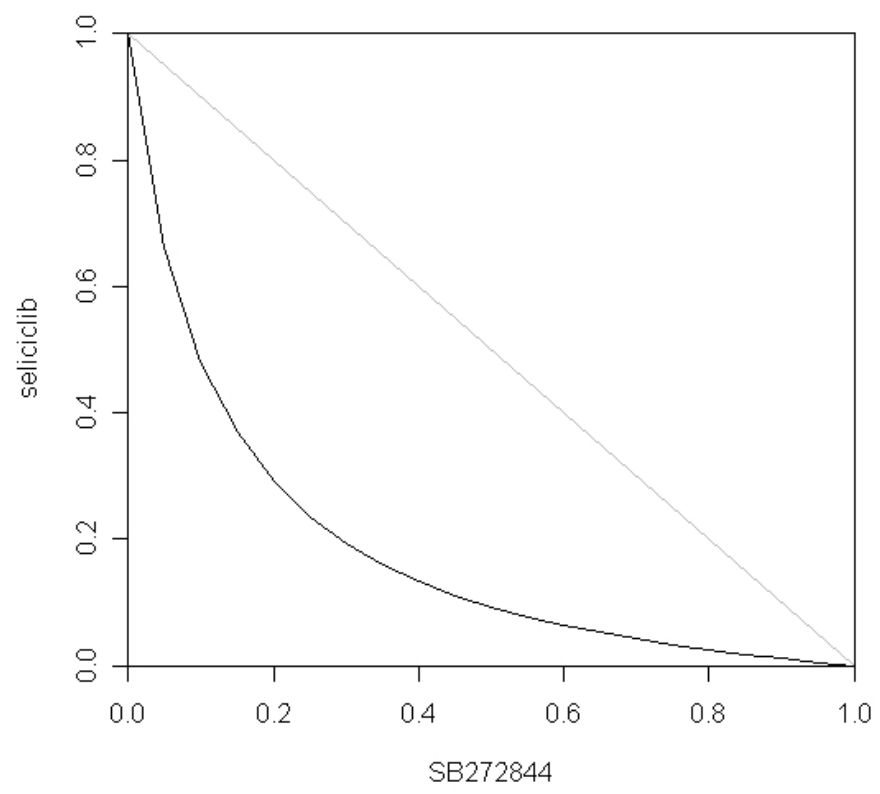

Figure 8. Isobol plot for the SB272844 + seliciclib combination. The diagonal shows the line of additivity. The concave shape of the isobol indicates synergy.

\section{Discussion}

The myeloN model captures the key qualitative features of neutrophil trafficking and activation, and of the response of these processes to infection and inflammation. $\mathrm{cAbl}$ appears to act as the primary control point for neutrophil activation, and in turn, through its effect on STAT transcription factors, activates multiple pathways. We have chosen to describe two of these in detail: the STAT5-mediated up-regulation of Mcl-1, which turns the normally transient tissue neutrophils into a much more long-lived cell population. Secondly, our model describes ROS production following neutrophil activation. This effect is driven by STAT3. Such ROS can arise from mitochondria, peroxisomes, or NADPH oxidase in the plasma membrane. In this paper we let mitochondrial ROS represent all three possible sources. ROS mediate the primary bactericidal function of neutrophils, but if they persist too long, they can cause serious oxidative damage to normal tissues. This tissue damage is a primary cause of morbidity in COPD and other inflammatory diseases.

A gatekeeper role of c-Abl is supported by the fact that its permanent activation results in malignant transformation. The Abelson murine leukaemia virus transforms murine lymphoblasts by expressing a constitutively activated form of the gene, v-Abl [34]. In humans, chronic myeloid leukaemia (CML) is caused by a chromosomal translocation that results in a fusion gene, Bcr-Abl, whose Abl domain is constitutively active [26]. These oncogenic forms of the Abelson gene appear to be sufficient for transformation. An inhibitor of the $A b l$ domain of Bcr-Abl, imatinib, gives high response rates, and long response durations in CML [29], reinforcing the critical role of the Abelson gene in neutrophil function.

The aetiology of COPD is complex, but there is general agreement that production of inflammatory cytokines, stimulated by inhaled irritants such as tobacco smoke, is the primary cause. The tissue injury caused by COPD is attributable to oxidative damage, and it has been suggested that ROS present in tobacco smoke, as well as ROS produced by activated neutrophils, are responsible. ROS are equally harmful, regardless of their source, and it is likely that anti-oxidants may be beneficial whatever the source of the ROS. However, abnormally high ROS production persists long after cessation of smoking, and in such cases, at least, the primary source must be internal rather than external. Our computational model of COPD assumes that $\mathrm{c}-\mathrm{Abl}$ is chronically activated, and this single factor is predicted to lead to all the other consequences: abnormally high numbers of tissue neutrophils, persistent 
activation of tissue neutrophils, elevated production of the anti-apoptotic protein Mcl-1, inhibition of neutrophil apoptosis, very high levels of ROS, represented here by electron leakage from the mitochondrial ETC, and resulting tissue injury.

The branched signalling pathway downstream from Bcr-Abl has interesting implications for the treatment of COPD. Since the damage to lung tissue in this disease is caused by ROS, it is reasonable to expect that antioxidants should have activity in this disease, and indeed the model predicts that this will be the case. However, the antioxidant effect, though providing symptomatic relief, does not address the causes of the problem, the continued activation of tissue neutrophils. Imatinib and seliciclib do cause depletion of activated tissue neutrophils, and the model predicts that they will be effective treatments for COPD. However, their effect is somewhat delayed, as the reactivation of apoptosis takes a few days to deplete the activated neutrophil population. Perhaps a combination of the two approaches will be superior to either used separately. The model suggests that the combined effects of ascorbate and seliciclib should be additive. Interestingly, the most striking synergy was predicted to result from the simultaneous triggering of tissue neutrophil apoptosis with seliciclib, and prevention of neutrophil trafficking from plasma into lung tissue, with SB272844.

The myeloN model thus provides a tool for rapid prediction of effective sites for drug intervention in COPD and other neutrophil-driven inflammatory conditions. Of course its predictions must be subjected to experimental verification, but the ability to explore a large number of possibilities in silico makes it possible to prioritise scarce experimental resources for those targets and drugs most likely to be successful. This is particularly the case for devising effective combination protocols.

\section{References}

1. Baggiolini M, Clark-Lewis I. Interleukin 8, a chemotactic and inflammatory cytokine. FEBS Lett 1992; 307: 97-101.

2. Utsumi T, Klostergaard J, Akimaru K, Edashige K, Sato EF, Utsumi K. Modulation of TNF-alpha priming and stimulationdependent superoxide generation in human neutrophils by protein kinase inhibitors. Arch Biochem Biophys 1992; 294: 271-278.

3. Schlatterer SD, Acker CM, Davies P. c-Abl in neurodegenerative disease. J Mol Neurosci 2011; 45: 445-452.

4. Nam S, Williams A, Vultur A, List A, Bhalla K, Smith D, Lee FY, Jove R. Dasatinib (BMS-354825) inhibits Stat5 signaling associated with apoptosis in chronic myelogenous leukemia cells. Mol Cancer Ther 2007; 6: 1400-1405.

5. Hancock JT. Cell Signalling (Third edition), p. 130. Oxford University Press 2010.

6. Kirchner T, Möller S, Klinger M, Solbach W, Laskay T, Behnen M. The impact of various reactive oxygen species on the formation of neutrophil extracellular traps. Mediators of Inflammation 2012; doi:10.1155/2012/849136.

7. MacNee W. Oxidants/antioxidants and chronic obstructive pulmonary disease: pathogenesis to therapy. Novartis Found
This is an inherently combinatorial problem, especially when multiple drugs, multiple dose levels, sequencedependent effects and varying degrees of drug selectivity are involved. Based upon the nine sites for inhibition included in the myeloN model, there are $(9 \times 8) / 2 !=36$ mechanistically distinct binary inhibitor combinations. Of these, we have so far only explored three, of which one was predicted to be moderately synergistic, one additive, and one highly synergistic. In future studies we plan to explore the remaining 33 binary combinations and the $(9 \times 8 \times 7) / 3 !=84$ possible three-drug combinations.

In some therapeutic areas, such as oncology [35] and cardiovascular disease [36] complex pharmacodynamic modelling has provided a tool for planning biomarker strategy - which biomarkers will correlate best with clinical outcome, which sampling strategy will have the greatest information content. The myeloN model of c-Abl signalling in COPD may be useful in identifying biomarkers of oxidative damage, and the effects of antioxidants that can guide the design of clinical use of antioxidants.

The myeloN model, in its present form, is a PD model: it relates drug concentrations to a PD effect. As such, it can model the effect of constant drug concentrations, as in in vitro experiments. To describe in vivo drug effects, it will be necessary to combine the myeloN model with a pharmacokinetic (PK) model that describes how the drug distributes within the body, and how its concentration changes with time. When validated against in vivo experimental data, including biomarker measurements, and combined with human PK data, these PK/PD models have the potential to be used for in silico clinical trials.

Acknowledgements: TR was supported by NCI ICBP NO 5U54CA149233-029689 and by 1RO1CA138858.

Symp 2001; 234: 169-185.

8. Valko M, Leibfritz D, Moncol J, Cronin MT, Mazur M, Telser J. Free radicals and antioxidants in normal physiological functions and human disease. Int J Biochem Cell Biol 2007; 39: 44-84.

9. Reddy SP. The antioxidant response element and oxidative stress modifiers in airway diseases. Curr Mol Med 2008; 8: 376-383.

10. Christóvão $\mathrm{C}$, Cristóvão $\mathrm{C}$. Evaluation of the oxidant and antioxidant balance in the pathogenesis of chronic obstructive pulmonary disease. Rev Port Pneumol 2013; 19(2): 70-75

11. Adcock IM, Caramori G. Kinase targets and inhibitors for the treatment of airway inflammatory diseases: the next generation of drugs for severe asthma and COPD? Biodrugs 2004; 18: 167180.

12. Rahman I, MacNee W. Antioxidant pharmacological therapies for COPD. Curr Opin Pharmacol 2012; 12: 256-265.

13. Rossi AG, Sawatsky DA, Walker A Ward C, Sheldrake TA, Riley NA et al. Cyclin-dependent kinase inhibitors enhance the resolution of inflammation by promoting inflammatory cell apoptosis. Nat Med 2006; 12: 1056-1064.

14. Milot E, Filep JG. Regulation of neutrophil survival/apoptosis by 
Mcl-1. The Scientific World Journal 2011; 11: 1948-1962.

15. Leitch AE, Riley NA, Sheldrake TA, Festa M, Fox S, Duffin, R et al. The cyclin-dependent kinase inhibitor R-roscovitine downregulates Mcl-1 to override pro-inflammatory signalling and drive neutrophil apoptosis. Eur J Immunol 2010; 40: 1127-1138.

16. Jackson RC, Barnett AL, McClue SJ, Green SR. Seliciclib, a cell-cycle modulator that acts through the inhibition of cyclindependent kinases. Expert Opin Drug Discov 2008; 3: 131-143.

17. MacCallum DE, Melville J, Frame S Watt K, Anderson S, Gianella-Borradori A et al. Seliciclib (CYC202, R-roscovitine) induces cell death in multiple myeloma cells by inhibition of RNA polymerase II-dependent transcription and down-regulation of Mcl-1. Cancer Res 2005; 65: 5399-5407.

18. Gilbert D, Fuss H, Gu X, Orton R, Robinson S, Vyshemirsky V et al. Computational methodologies for modelling, analysis and simulation of signalling networks. Brief Bioinform 2006; 7(4): 339-353.

19. Terry AJ, Chaplain MAJ. Spatio-temporal modelling of the NF$\kappa \mathrm{B}$ intracellular signalling pathway: The roles of diffusion, active transport, and cell geometry. $J$ Theor Biol 2011; 290: 7-26.

20. Rubinow SI, Lebowitz JL. A mathematical model of neutrophil production and control in normal man. J Math Biol 1975; 1: 187225.

21. Zhuge C, Lei J, Mackey MC. Neutrophil dynamics in response to chemotherapy and G-CSF. J Theor Biol 2012; 293: 111-120.

22. Brooks G, Provencher G, Lei J, Mackey MC. Neutrophil dynamics after chemotherapy and G-CSF: the role of pharmacokinetics in shaping the response. $J$ Theor Biol 2012; 315: 97-109

23. Scholz M, Schirm S, Wetzler M, Engel C, Loeffler M. Pharmacokinetic and-dynamic modelling of G-CSF derivatives in humans. Theor Biol Med Model 2012; 9: 32-60.

24. Harrap KR, Jackson RC. Some biochemical aspects of leukaemias. Leukocyte glutathione metabolism in chronic granulocytic leukaemia. Eur J Cancer 1969; 5: 61-67.

25. www.morphosys.com/pipeline March 2013.

26. Shtivelman E, Lifshitz B, Gale RP, Canaani E. Fused transcript of abl and bcr genes in chronic myelogenous leukaemia. Nature
1985; 315: 550-554.

27. Carella AM, Daley GQ, Eaves CJ,Goldman JM, Rudier H. Chronic Myeloid Leukaemia: Biology and Treatment. London: Martin Dunitz (2001).

28. Cortes J, Deinger M. Chronic Myeloid Leukemia. New York: Informa, 2007.

29. Druker BJ, Talpaz M, Resta DJ, Peng B, Buchdunger E, Ford JM et al. Efficacy and safety of a specific inhibitor of the BCR-ABL tyrosine kinase in chronic myeloid leukemia. N Engl J Med 2001; 344: 1031-1037.

30. Deininger M, Buchdunger E, Druker BJ. The development of imatinib as a therapeutic agent for CML. Blood 2005; 105: 26402653.

31. Greco WR, Park HS, Rustum YM. Application of a new approach for the quantitation of drug synergism to the combination of cisdiamminedichloroplatinum and 1- $\beta$-D-arabinofuranosylcytosine. Cancer Res 1990; 50: 5318-5327.

32. Chou TC, Talalay P. Quantitative analysis of dose-effect relationships: the combined effects of multiple drugs or enzyme inhibitors. Adv Enzyme Reg 1984; 22: 27-55.

33. Greco WR, Bravo G, Parsons JC. The search for synergy: a critical review from a response surface perspective. Pharmacol Rev 1995; 47: 331-385.

34. Goff SP. The Abelson murine leukemia virus oncogene. Proc Soc Exp Biol Med 1985; 179: 403-412.

35. Jackson RC. Pharmacodynamic modelling of biomarker data in oncology. ISRN Pharmacology 2012; 2012: 590626. Doi:10.5402/2012/590626.

36. Chan JR, Vuillaume G, Bever C, Lebrun S, LietzM, Steffen Y et al. The apoe-/- mouse PhysioLab ${ }^{\circledR}$ platform: a validated physiologically-based mathematical model of atherosclerotic plaque progression in the apoe-/- mouse. Biodiscovery 2012; 3:2.

37. Jackson RC. The Theoretical Foundations of Cancer Chemotherapy Introduced by Computer Models. New York, Academic Press, 1992.

38. Venables WN, Smith DM. An Introduction to R. Bristol: Network Theory Ltd, 2005. 